\title{
Philosophy of Technology in Affective Computing and Social Network Analysis
}

Moreno Luis, Mgr. in Engineering ${ }^{1}$, Pomares Alexandra, Dr. in Engineering ${ }^{1}$, and Salinas Martha, Dr. in Education ${ }^{2}$

${ }^{1}$ Pontificia Universidad Javeriana, Colombia, morenoluis@javeriana.edu.co, pomares@javeriana.edu.co

${ }^{2}$ Minuto de Dios University Corporation, Colombia, msalinas@uniminuto.edu

\begin{abstract}
The paper assumes Philosophy of Technology importance in Affective Computing and Social Network Analysis from three perspectives: challenges generated by changes in relationships stablished in social networks, contemporary interactions nature between artifacts, humans and environments throughout concepts as meaning and experience and applying data mining to enhance information comprehension, reflection and influence in contemporary knowledge societies. Through a flexible methodology of comparing concepts presented in literature to expert's viewpoints, it outlines some of the key ethical issues around analysis of social interactions shared in the web.
\end{abstract}

Keywords-- affective computing, philosophy of technology, social network analysis, technology and society

\section{INTRODUCTION}

Affective computing involves multidisciplinary knowledge of psychology, cognition, physiology, and computer science. What it looks for is the allocation of human capacities to the computers such us observation, interpretation and generation of characteristics associated to the subject. The analysis of "private states" that is, mental or emotional states that cannot be directly observed or verified, which include opinions, emotions, valuations, speculations, feelings[1], [2] is directly related to the capacity of technology to observe, interpret and detect the expression of such manifestations of subjectivity in human communication.

It has been shown the need to define a series of semantic properties to produce collective subjectivity analysis in social networks from data mining, for which the definition, evolution, measurements and methods of two major chapters of the computing sciences have been addressed: social network analysis (SNA) and subjectivity analysis. The latter is understood as the task of extracting, processing and analyzing "private states" exposed in human communication on the web. The subjectivity analysis is part of the previously described field of artificial intelligence called Affective Computing [3].

The need for the philosophy of technology in this area can be addressed from three angles. The first, as expressed by [4] regarding the challenges brought about by changes in community relationships that are established in social networks giving rise to more open and less hierarchical structures, and patterns of dynamic relationships in society, With the consequent response to questions about how technology creates new networks of control and their potential involvement in moral discourses and practices. In this sense, [5] introduces the concept of "social affordances", which offers a less deterministic sense of the opportunities and constraints that technology provides.

The second edge from which the philosophy of technology is visible consists of the nature of contemporary interactions between artifacts, humans, and environments through concepts such as meaning and user experience. The use of social networks like Twitter is increased with the progressive increase of such interactions. Thus, two philosophies of contemporary technology merit an important look: Albert Borgmann 's notion of the device paradigm, and Don Ihde' s notion of non neutrality of technology - mediated experience.[6].

Finally, we propose the importance of the philosophy of technology in a third area related to data mining for information literacy. This aspect is based on [7], on the capacity of the application of data mining to strengthen the understanding, reflection and influence of information and activities of production and consumption of information in contemporary knowledge societies. Data mining is conceived as a means to promote information literacy from two sub competencies: critical data literacy and private literacy, both among debates in the philosophy of technology over criticisms of the instrumental reasons for the use of Private information and the possibilities and nature of artificial intelligence.

\section{StATE OF THE ART IN AFFECTIVE COMPUTING}

Affective computing is about assigning computers human capacities such as observation, interpretation and generation of affect characteristics. It is an important topic for the harmonious interaction between human and computer, since it contributes to the increase of the quality of the communication between them and to the improvement of the computational intelligence.

Research on affect or emotion dates back to the nineteenth century, although traditionally the concept of "affection" was seldom associated with lifeless machines, and was studied by psychologists. However, recently the characteristics associated with affection were captured and processed through computers. Affective computing builds an "affection model" based on the varied information captured through various mechanisms and builds a personalized computer system with the ability to perceive, interpret human feelings and in the most advanced cases giving us intelligent, sensitive and friendly answers[3].

The technologies registered in the literature on affective computing have been developed in order to capture affective information and model it in some way. Since people express 
affection through a series of facial expressions, body movements, gestures, voice behaviors, psychological signals, and linguistic expressions, technologies are classified as emotional speech processing, recognition and generation of facial expressions, multimodal movement systems and body gestures, text mining, among others.

Within the mentioned framework of affection expressions, we find the notion of subjectivity present in linguistic literature in various ways, all of them referring to the systematic forms in which the speaking subject manifests itself in language. In the linguistic field, one of the most cited authors is Traugott [8][10], recognized for her notion of subjectivity as a semantic property of linguistic forms (morphemes or clusters of morphemes), that is, there are words or groups of words that have an inherently more objective meaning because they refer to "things" in the world around us: objects, events, and their properties, while others are inherently more subjective because they refer, for example, to evaluations that the subject makes about things in the world.

Information retrieval, information extraction, and question - answer techniques are some of the goals of text mining. Recently, however, there has been a marked interest in exploring what Wiebe [11]-[14] defines as the "private states" term that encompasses feelings, opinions, emotions, valuations, beliefs and speculations, giving rise to a new branch of PLN called Subjectivity Analysis, defined as the application of automatic tools and techniques for extracting opinions, emotions, feelings and, in general, from any linguistic expression of "private states" [12], [15]-[17].

\section{OBJECTIVES AND STUDY METHODOLOGY}

Theoretical analysis, reviewing concepts in the existing literature about the three mentioned perspectives for incorporating Philosophy of Technology in Affective Computing and Social Network Analysis and comparison of answers to semi-structured interviews developed with five experts in social network analysis and data mining.

Being the main purpose of the study to determine the ethical and moral implications associated with the analysis of social relationships established on the web and the treatment of the private information available in social networks, the results of studies associated with the way in which technology creates new control networks and their potential involvement in moral discourses and practices were described. Next, we sought to write the ethical and moral implications associated with the nature of contemporary interactions between artifacts, humans and environments, and finally, we identified the emerging ethical considerations in the development of competencies in information literacy in the context of data mining.

A methodology for documentary review of primary studies was developed for the identification of studies associated with the general objective of the research around the following constructs, in English and Spanish: "ethics" and "social network analysis", "Private information "and" social network analysis ".

In addition, it was considered necessary to interview five experts in Social Network Analysis and in Data Mining to know the ethical positions in practice in these disciplines. The information was organized in matrices for the analysis of the results around each of the proposed objectives.

\section{RESULTS}

The information obtained through the systematic review of the documents, along with the results of interviews with five experts in Social Network Analysis and Data Mining, was organized around the three perspectives that motivated the study. Main findings are recorded below.

A. Creation of new networks of control through technology and its potential involvement in moral discourses and practices.

According to [4], social network analysis has been used to explain the structures of relationships and online communities, described in terms of people's social use of the internet, in a freeway, to maintain relationships, establish links, share information, etc. Modern media are digitally encoded and are based on numerical representations, which makes them modular and maintains separate identities. This feature makes them vulnerable to being automated as operations, stored and manipulated separately, partly removing the human element.

The new technologies are variable since they allow multiple versions and involve transcoding, that is, the translation of data and products into other formats. Each of these features makes the new media dynamic, malleable, programmable and capable of being personalized, while allowing collective construction as well as highly individualized use. These characteristics highlight a change not only in the structure of technology but also in the way we conceive communication.

Manovich[18] states that the transcoding of new media allows two different layers to be observed in the communication process: the "computational layer" and the "cultural layer", each influenced by the other, resulting in the nature and characteristics of the computational technology of forming our cultural understandings on the processes that they facilitate and vice versa. The result is a mixture of computational and human meanings of the traditional forms in which human culture modeled the world and own terms of the computer to represent it.

For the author, the new media impel a "meta-realism" in which the user is endowed with the power to discern, deconstruct and control the reality or "illusion" with which he or she presents, in contrast to the "old-style realism" of

16 ${ }^{\text {th }}$ LACCEI International Multi-Conference for Engineering, Education, and Technology: "Innovation in Education and Inclusion", 19-21 July 2018, Lima, United States. 
traditional media in which the user can simply accept or reject the illusion or reality presented. Meta - realism is based on selfcriticism, people are taught not to take what is given literally, but are encouraged to investigate, experiment and change. While traditional media provide a "window into the world," new media offer a "dashboard" enabling the creation of alternative realities.

According to [18], [19], the new media are transformed from a set of prescribed narratives to a changeable and interactive configuration. New media empower people to manipulate structures and meanings. On this view, Campbell [4] argues that traditional communities were characterized by fixed boundaries and established connections with homogeneous, small-scale, structurally regulated and geographically defined relationships. Modern society, on the other hand, has been defined by its fluid boundaries, changing interactions, and specialized, diverse, large-scale associations that are relationally defined.

The analysis of social networks has been used as a way of directing and studying these changes in the conception and configuration of the communities. Since computational culture promotes individual selection, malleability, and dynamic interaction, modern society facilitates the configuration of communities based on specialized association and fluid boundaries that are personally regulated and based on selected associations.

The security and control of inclusive communities has given rise to the opportunity and vulnerability of interconnected individualism ([20], which denotes a shift towards life in networks governed by individual selection rather than in collaborative groups. Internet users have made their own home in self-selected networks as members that gather around a common topic in various online forums. Online communities represent a paradox, presenting both the focus on the relationship and the promotion of individual action and personal control.

In this context, the interviewed experts argue that the implications of these new configurations on moral discourses and practices are essentially the same implications of the interconnected individualism proposed by [20], and this is how heterogeneity and new dimensions of control within the interconnected networks, gives rise to the emergence of power exercises marked by influence, closeness, interconnection, and the centrality of the different actors in the networks and by the conditions and characteristics of the links which they establish. They validate from their experience the paradox of personal control, malleability, dynamic interaction and the influence of the computational layer.

Experts agree that human interactions in community have changed in terms of scale but have preserved the essence of the human condition in which the control of the information that is exposed is still in the user and that although the technology facilitates access, human control over technology is still in place. It is in the cultural layer that underlie the ethical and moral implications that determine the capacity of generation and penetration of control networks. On the other hand, communities established now online, which do not differ from what constituted the communities of small towns or cities a few centuries ago, are exposed to what one of the experts calls "false contact" and "real isolation "As long as the personalization of the relationship with the respective consequences that this condition brings with it, is eliminated from the cultural layer.

\section{B. Ethical and moral implications associated with the nature of contemporary interactions between artifacts, humans and environments.}

The use of social networks such as Twitter is increased with the progressive increase of contemporary interactions between artifacts, humans and environments through concepts such as meaning and user experience Albert Borgmann (2000), proposes the device paradigm defined as the distinctive conjunction of an available good and a sophisticated and impenetrable machinery, based on the principle that growing and vigorous consumption is the main indicator of a prosperous and self-confident community.

The promise of technology according to the author has become a fruitful and beneficial reality, although it has given rise to technological devices increasingly refined, effective and complicated, which makes it necessary to watch over the principle of symmetry between reality and humanity that the quality of the human condition and the quality of the material environment tend towards one and the same level.

According to [21], human evolution is the great spectacle of an animal that opens itself to higher levels and dimensions of reality and what the theory of evolution tells us is that if that symmetry fails at any level, species tend to disappear. The human company of technology began to dislocate the natural symmetry when it separated its pleasures from its context of effort and in the process, the factors that it used to attenuate our desires were surpassed and eliminated and our desires began to get uncontrolled.

Paradigmatic consumption is not a crime or a sin in the traditional sense but is intrinsically decoupling. If it dominates our pleasure, it weakens our physical and mental vigor and displaces or destroys things and practices that must be taken care of in return for gaining security, ease, space and time thanks to the incorporation in our lives of all kinds of technological devices[21]. The expert in social network analysis in the field of administration interviewed states that the use of technological devices has had a significant impact on social behavior and has given rise to new ways of fun, but in turn has relegated practices that will necessarily affect human and social evolution.

For [6], analog and mechanical technologies have rapidly been enhanced with digital capabilities such as network performance capabilities, "intelligent" behavior and interactive 
features, as well as a wide range of new penetrating and generalized technologies such as Bluetooth, Wireless LAN, ANT, 3G, 4G are enabling these digital devices to communicate with each other, to create ad-hoc networks, to negotiate offers, and to exchange information, leaving us originally known as users to one side. From this perspective of human-computer interaction (IHC), these trends and their associated questions and uncertainties are mainly focused on what is called the user experience.

The data mining experts interviewed say that the nature of contemporary interactions between artifacts, humans and environments has been transformed at the same speed of technological advances and has generated equally complex consumer attitudes. The devices that we acquire are not only easier to dispose of, because of their components but also because of the technological scope that the new device offers us. In turn, the interaction between devices has made public an immense amount of personal information that although it is an input for multiple investigations, is also impregnated with the ethical and moral character of the exhibition of the privacy and the use that of the information staff is made.

They coincide in that there are two possible glances at the phenomenon of propagation of artifacts and unlimited technological advances: the apocalyptic vision in which the human being is absorbed and dominated by the technology and the vision that humanizes the technology as it configures it as an unprecedented support for the human capacity over which the human remains in control and moral and ethical responsibility.

C. Emerging ethical considerations in the development of competencies in information literacy in the context of data mining.

Berendt[7] proposes the application of data mining as a means to help people to better understand information and information production and consumption activities that surround current knowledge societies. According to the author, data mining is a means to promote information literacy and specifically critical literacy, supported by the principles of a culture of democratic behavior in the informational environment of society.

Information literacy consists of the ability of individuals not only to use information and information technologies effectively and adapt it to their constant changes but also to think critically and comprehensively about the information and information society. On the other hand, critical literacy consists in the development of habits of deep understanding of meaning, causes, social context, ideology, and all the possible characteristics that surround information. It is defined as the ability to critically assess human, intellectual and social strengths and weaknesses, the potentials and limits, benefits and costs of information technologies [7].
The main ethical considerations from data mining, in general, and text mining in particular are associated with the ability to analyze the information obtained. While the emergence of Web 2.0 and subsequent advances in technology have given rise not only to increasingly advanced techniques but to an immense amount of information available, the treatment of these data must be based on ethical principles that avoid transgression of the privacy and the affectation of the other, since they are framed in the capacity of adequate use of the information. Such is the case of text mining in social networks which is one way of analyzing the content of the information that is shared on the Web.

The analysis of contents in social networks is mainly addressed through data mining, text mining, multimedia mining and stream mining in social networks, advances that correspond to the conditions determined by the increase in technological possibilities, as well as in the provision of abundant content. For [22], the field of social networks has become the victim of their own success. While it is possible to calculate the value of a person's network of friends, but also to obtain maps of terrorist and criminal networks. Many organizations attempt to improve their efficiency through sociometrical analyzes, epidemiology has benefited from the information provided by scatter analysis methods, structures of national leaders and decision makers have been studied and the amount of information analysis applications available on the Web is increasing.

To the same extent, ethical issues vary between simplicity and complexity. In standard practices of social science research, anonymity and confidentiality are guaranteed to participants, informants, and subjects of experiments and observations on a routine basis. On the other hand, social network analysts have public information and connections between actors are evidenced even without knowledge of the same actors. Ethics lies in making assertions about connections, centrality, and power when the reliability and accuracy of data sources can be challenged even they are publicly available. An example could be the ethical consideration that exists in making visible the connection of a particular individual that can bring negative consequences for that person.

According to [22], many ethics committees conclude that any way to identify individual participants in a study is illegitimate and unethical, which makes privacy an absolute right, whereby collecting personal data in networks is noxious regardless of any potential benefit of the research. This situation becomes even more obscure when the studies do not go through any ethics committee, so the concern about "legitimate" network researchers is the same concern about individual rights to privacy.

The experts interviewed are researchers from prestigious universities and their studies have been validated by ethics committees, and they agree in their position facing the current debates in the philosophy of technology on the criticisms of the

16 $^{\text {th }}$ LACCEI International Multi-Conference for Engineering, Education, and Technology: "Innovation in Education and 
instrumental reasons of the use of private information about that private data cannot be disclosed without the knowledge and authorization of the participants. However, availability in public APIs has a high risk of improper handling of information, especially when it is not used for research purposes.

\section{CONCLUSIONS}

The evolution in the analysis of social networks, as well as in the use of data mining techniques for the analysis of individual and collective subjectivity has recorded important research advances. However, most of the work focuses on the social impacts of such technologies, but not on the related ethical dimensions, especially with problems associated with privacy. The main ethical issues present in the interaction in social networks refer to the rights to privacy and dependence present in networks, such as building community rules in social networks, intellectual property rights, intrusion to private companies, among others.

The documentary review and the interviews with the experts allow us to conclude that the capacity of technologies to deal with the phenomena of affectivity and the detection of subjectivity oscillates between $40 \%$ and $50 \%$ understanding that the challenges still not addressed and the magnitude of the missing ones have not been completely determined. On the other hand, it is considered that the research supported in the analysis of the personal information available on the network, should take into account ethical considerations such as the exposed in the next paragraphs.

The implications of interconnected individualism, heterogeneity and new dimensions of control within interconnected networks, lead to the emergence of power exercises marked by influence, closeness, interconnection, and the centrality of the different actors in the networks and by the conditions and characteristics of the links they establish.

The main challenges that come with the changes in community relations established in social networks are for experts, mass and anonymity, relationships with the environment in the presence of artifacts and the possibility of losing the ability to relate to others or to generate dependencies on technology to ensure happiness.

Networking capabilities, "intelligent" behavior and interactive features, as well as the wide range of new and penetrating technologies such as Bluetooth, Wireless LAN, ANT, 3G, 4G are enabling communication between a wide variety of devices and digital artifacts, to create ad-hoc networks, negotiate offers, and exchange information where it is necessary to redefine the role of the user as an agent endowed with the capabilities to determine the scope of use and to control it.

The capacity of the application of data mining to strengthen the understanding, reflection and influence of information and activities of production and consumption of information in contemporary knowledge societies constitutes a historical contribution that, however, is mediated by the current debates on the management of the privacy of information and the use of data available on the web.

\section{ACKNOWLEDGMENT}

This research was carried out by the Center of Excellence and Appropriation in Big Data and Data Analytics (CAOBA). It is being led by the Pontificia Universidad Javeriana Colombia and it was funded by the Ministry of Information Technologies and Telecommunications of the Republic of Colombia (MinTIC) through the Colombian Administrative Department of Science, Technology and Innovation (COLCIENCIAS) within contract No. FP44842-anex46-2015. Authors also thanks UNIMINUTO for the support in developing the study.

\section{REFERENCES}

[1] A. Montoyo, P. Martínez-Barco, y A. Balahur, «Subjectivity and sentiment analysis: An overview of the current state of the area and envisaged developments», en Decision Support Systems, 2012, vol. 53, n. ${ }^{\circ}$ 4, pp. 675679.

[2] T. Wilson, P. Hoffmann, S. Somasundaran, J. Kessler, J. Wiebe, Y. Choi, C. Cardie, E. Riloff, y S. Patwardhan, «OpinionFinder: A system for subjectivity analysis», en October, 2005, n. ${ }^{\circ}$ October, pp. 34-35.

[3] J. Tao y T. Tan, «Affective computing: A review», en International Conference on Affective computing and intelligent interaction, 2005, pp. 981995.

[4] H. Campbell, «Challenges created by online religious networks», $J$. Media Relig., vol. 3, n. ${ }^{\circ}$ 2, pp. 81-99, 2004.

[5] E. Bradner, «Social affordances of computer-mediated communication technology: understanding adoption», en CHI'01 Extended Abstracts on Human Factors in Computing Systems, 2001, pp. 67-68.

[6] D. Fallman, «The new good: exploring the potential of philosophy of technology to contribute to human-computer interaction», en Proceedings of the SIGCHI conference on human factors in computing systems, 2011, pp. 1051-1060.

[7] B. Berendt, «Data mining for information literacy», en Data Mining: Foundations and Intelligent Paradigms, Springer, 2012, pp. 265-297.

[8] E. C. Traugott, «On the rise of epistemic meanings in English: An example of subjectification in semantic change», Language (Baltim)., pp. 3155,1989

[9] E. C. Traugott y R. B. Dasher, Regularity in Semantic Change. Cambridge University Press, 2001

[10] E. Traugott, «Linguistic Society of America Linguistic Society of America», vol. 44, n. ${ }^{\circ}$ 2, pp. 267-273, 2011.

[11] J. Wiebe, E. Breck, C. Buckley, y C. Cardie, «Recognizing and Organizing Opinions Expressed in the World Press.», Work. Notes - New Dir. Quest. Answering (AAAI Spring Symp. Ser., pp. 12-19, 2003.

[12] C. Banea, R. Mihalcea, y J. Wiebe, «Sense-level subjectivity in a multilingual setting», en Computer Speech and Language, 2014, vol. 28, n. ${ }^{\circ}$, pp. 7-19.

[13] J. Wiebe y E. Riloff, «Creating subjective and objective sentence classifiers from unannotated texts», en Computational Linguistics and Intelligent Text Processing, 2005, vol. 3406, pp. 486-497.

[14] J. Wiebe, «Instructions for Annotating Opinions in Newspaper Articles», 2002.

[15] C. Banea, Y. Choi, L. Deng, S. Hassan, M. Mohler, B. Yang, C. Cardie, R. Mihalcea, y J. Wiebe, «CPN-CORE: A Text Semantic Similarity System Infused with Opinion Knowledge», en Second Joint Conference on Lexical 
and Computational Semantics (*SEM), 2013, pp. 221-228.

[16] C. Banea, J. Wiebe, y R. Mihalcea, «A Bootstrapping method for building subjectivity lexicons for languages with scarce resources», en Proceedings of the Language Resources Evaluation Conference (LREC), 2008, pp. 2764-2767.

[17] C. Banea, R. Mihalcea, J. Wiebe, y S. Hassan, «Multilingual Subjectivity Analysis Using Machine Translation», en Computational Linguistics, 2008, n. ${ }^{\circ}$ October, pp. 127-135.

[18] L. Manovich, «Post-media aesthetics», Transmedia Frict. Digit. Arts, Humanit., 2001.

[19] L. Manovich, «The Language of New Media». The MIT Press, Cambridge and London, 2001.

[20] B. Wellman, J. Boase, y W. Chen, «The networked nature of community: Online and offline», It Soc., vol. 1, n. ${ }^{\circ}$ 1, pp. 151-165, 2002.

[21] A. Borgmann, «The moral complexion of consumption», J. Consum. Res., vol. 26, n. ${ }^{\circ}$ 4, pp. 418-422, 2000.

[22] C. Kadushin, «Who benefits from network analysis: ethics of social network research», Soc. Networks, vol. 27, n.o 2, pp. 139-153, 2005.

$1^{\text {th }}$ LACCEI International Multi-Conference for Engineering, Education, and Technology: "Innovation in Education and Inclusion", 19-21 July 2018, Lima, United States. 